\title{
CONTROL PI ADAPTATIVO POR GANANCIA PROGRAMADA DEL NIVEL DE UN TANQUE DE SECCIÓN TRAPEZOIDAL
}

\author{
Manuel Lara Ortiz, Juan Garrido Jurado, Mario L. Ruz Ruiz*, Francisco Vázquez Serrano \\ Dpto. de Informática y Análisis Numérico, *Dpto. de Mecánica, Universidad de Córdoba, Campus de \\ Rabanales, Edificio Leonardo Da Vinci, Córdoba, España, \\ e-mail: p12laorm@uco.es, juan.garrido@uco.es,mario.ruz@uco.es, fvazquez@uco.es
}

\begin{abstract}
Resumen
Este trabajo aborda el problema del control de nivel de un tanque de sección trapezoidal. Este proceso es un sistema no lineal cuya dinámica varía según el punto de operación. Se propone una estructura de control adaptativo a través de un controlador PI por ganancia programada de fácil implementación. Dicho controlador se ajusta utilizando métodos en el dominio de la frecuencia en varios modelos linealizados del sistema no lineal en torno a diferentes puntos de trabajo. Los resultados en simulación son contrastados con los experimentales mostrando una buena concordancia y una buena respuesta del sistema.
\end{abstract}

Palabras Clave: control adaptativo, control PI, ganancia programada.

\section{INTRODUCCIÓN}

La regulación de flujo, temperatura, presión y nivel es una tarea de gran importancia en el funcionamiento de la mayoría de los procesos industriales. Los sistemas de control de nivel en tanques de líquidos aparecen en muchas industrias, por ejemplo, en el stock de las materias primas en la fabricación de productos químicos, el proceso de mezcla de materias primas en los procesos de litificación, y la reacción de productos de salida en la tecnología bioquímica. Los controles de nivel tienen como finalidad garantizar el nivel del líquido en un rango de variación preestablecido. Una de las principales dificultades que presenta es el tipo de dinámica que gobierna tales sistemas, la cual suele ser no lineal, como la variable de resistencia al flujo y la relación de nivel de flujo. Por lo tanto, es difícil realizar un control de alta precisión mediante el uso de métodos de control lineal. En el control de nivel en depósitos éste es un factor importante que considerar al definir esquemas de control. Existen tanques de base plana, ya sean cuadrados o cilíndricos que presentan un escenario más favorable al ser regidos por dinámicas de tipo lineal. Sin embargo, este tipo de tanques no son los más utilizados en la industria, ya que no garantizan un drenaje completo de los fluidos que contienen.

Además del interés industrial, no menos relevante es el académico por los sistemas no lineales, y más aún con aquellos que disponen de dinámicas relativamente rápidas y muy visuales, como lo son las hidráulicas, aunque el presente caso sea llevado de forma forzada al extremo, añadiendo al depósito una sección no cuadrangular.

Existen diversos trabajos que analizan el control de nivel de un tanque como proceso no lineal. Se han propuesto formas de modelado fenomenológico y control con estrategias básicas como control PI [2], [7]. Además, se han desarrollado técnicas de control por modelo interno [14] y controladores PID basados en este esquema [12]. Igualmente, para poder afrontar el problema que conlleva la no linealidad del proceso, se ha recurrido a técnicas más avanzadas de control, como por ejemplo control predictivo [9], [15], técnicas de control no lineales como Passivity Based Control (PBC) [4], o técnicas de computación evolutiva mediante algoritmos genéticos para la sintonización de los parámetros del controlador de nivel minimizando una cierta función objetivo [13].

A la hora de realizar diseños de controladores para sistemas no lineales, uno de los procedimientos más utilizados es el que consiste en linealizar las ecuaciones que modelan el sistema, realizando una expansión por series de Taylor alrededor de un punto nominal de operación. El problema aparece cuando el rango de variación de las condiciones de operación es amplio, ya que un controlador lineal no garantiza un adecuado funcionamiento del sistema. En estos casos puede ser necesario diseñar un controlador no lineal.

El control por ganancia programada (gain scheduling) es una técnica de control no lineal que ha sido aplicada con éxito en diferentes procesos como por ejemplo en aviación, en el control de vuelo y pilotos automáticos [8] o en misiles para compensar los cambios en la altitud y la velocidad [11]. En automoción, la programación de la ganancia de los controladores de motores comenzó en conjunto con el uso de un control de mezcla de aire/combustible 
basado en microprocesadores y en respuesta a los imperativos duales de mejorar el ahorro de combustible y la reducción de las emisiones de escape. Ejemplos son el uso de catalizadores, los cuales requieren de un control preciso de la relación aire/combustible [5]. En 1978, Ford propuso la incorporación de inyección de combustible y control de aire/combustible por retroalimentación exclusiva con la ganancia del integrador programada para la velocidad del motor [3].

En este trabajo se presentan una estrategia de control PI adaptativo por ganancia programada del nivel de un tanque de sección trapezoidal cuya implementación es muy sencilla. Para esto se obtiene un modelo no lineal del proceso y se linealiza en torno a tres puntos de operación en base a la altura del líquido. Luego, se sintonizan los parámetros del controlador PI para el mismo margen de fase, de forma que el controlador se comporte eficientemente en todo el rango de operación. El diseño se verifica tanto en simulación como experimentalmente.

Este trabajo está organizado como sigue: la Sección II incluye una descripción detallada de los componentes de la planta y el modelado del tanque trapezoidal. La Sección III detalla la estrategia de control adaptativo, su ajuste e implementación. En la Sección IV se muestran los resultados obtenidos tanto en simulación como con la planta real y la Sección V presenta las conclusiones del trabajo.

\section{TANQUE DE TRAPEZOIDAL}

\subsection{PLANTA EXPERIMENTAL}

En la Figura 1 se muestra un esquema de la planta experimental, la cual se encuentra en el Laboratorio de Control de Procesos de la Universidad de Córdoba. Consta de los siguientes elementos:

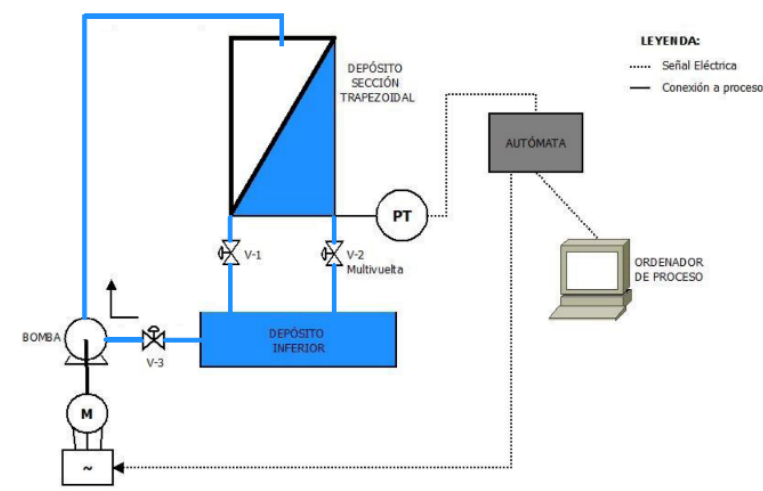

Figura 1: Esquema del sistema.

- Un depósito inferior del cual se toma el agua y al que vuelve.
- Un depósito de metacrilato al que se ha dotado de una sección trapezoidal utilizando unas planchas de corcho plastificado. Es en este tanque donde se controla el nivel de agua.

- Una bomba que toma agua del depósito inferior y la envía al tanque trapezoidal.

- Tubos y válvulas para las conexiones hidráulicas. En la parte inferior del tanque trapezoidal hay dos válvulas abiertas por donde el agua cae al depósito inferior.

- Un sensor de presión por diafragma de la marca Wika para medir el nivel de agua del tanque trapezoidal. Su rango es de 0-0.1 bar, lo que en columna de agua equivale aproximadamente a 0-100 $\mathrm{cm}$.

- Un variador de Siemens para modificar la velocidad de la bomba.

- Un sistema de control distribuido de Opto 22 formado por un sistema de E/S al que se conectan el sensor de nivel y el variador, y un controlador LCM4 en el que se pueden implementar las diferentes estrategias de control de caudal. Dicho sistema, además, está conectado a un PC desde donde se programan las estrategias de control y donde hay un SCADA diseñado para la planta.

En la figura 2 se muestra una foto de la planta y el cuadro de control:

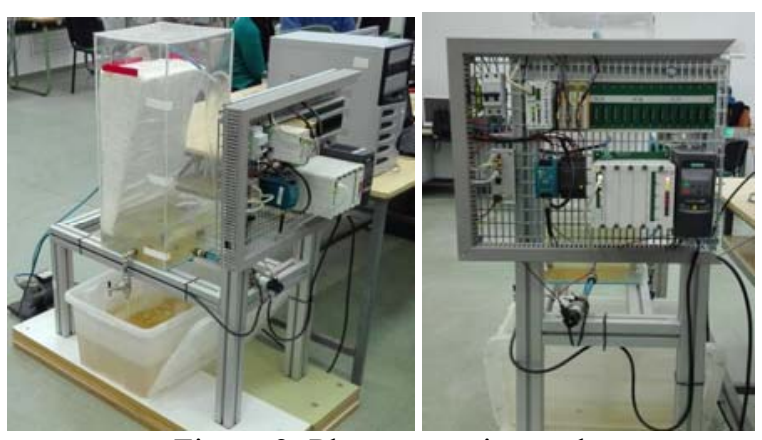

Figura 2: Planta experimental

\subsection{MODELADO DEL SISTEMA}

El modelo físico del sistema de la planta trapezoidal se obtiene partiendo de la ecuación básica de conservación de la masa, donde la derivada del volumen de agua respecto al tiempo es igual al caudal de entrada menos el caudal de salida (1). En los siguientes subapartados, se detalla cómo se modelan y obtienen cada uno de estos tres términos.

$$
\dot{\mathrm{V}}=\mathrm{q}_{\mathrm{e}}-\mathrm{q}_{\mathrm{s}}
$$

\subsubsection{Derivada del volumen}

Dado que la sección lateral del tanque es trapezoidal, para una altura dada $h$, el volumen de agua en el tanque será el área de dicho trapecio por el largo constante del tanque (de $29 \mathrm{~cm}$ ), esto es, 
$\mathrm{V}(\mathrm{h})=29 \cdot \mathrm{A}(\mathrm{h}) . \mathrm{El}$ área de dicho trapecio depende de la altura $\mathrm{h}$ y del ancho $\mathrm{x}$ del tanque a dicha altura:

$$
\mathrm{A}(\mathrm{h})=\mathrm{h} \frac{(\mathrm{x} 0+\mathrm{x})}{2}
$$

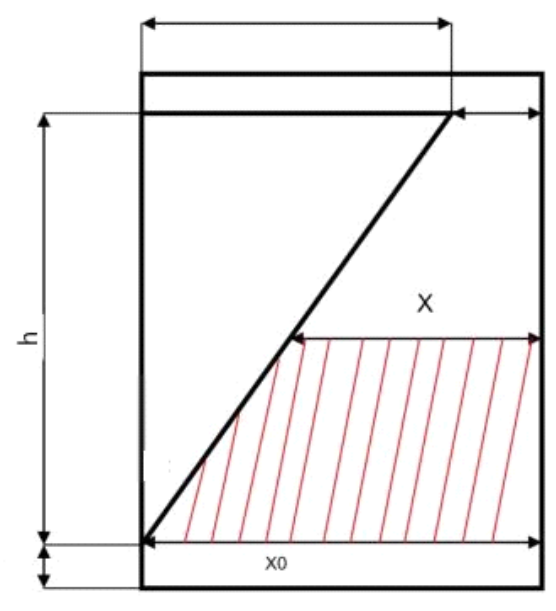

Figura 3: Esquema lateral del tanque trapezoidal

El ancho del tanque $\mathrm{x}$ también depende de la altura $\mathrm{h}$ según relación lineal. Conocido el ancho en dos alturas diferentes (y siendo las alturas medidas con el sensor de presión) se obtiene la recta $\mathrm{x}(\mathrm{h})=-$ $0.2867 \cdot h+25.38$. Así pues, la expresión final del volumen $\left(\mathrm{en}^{3}\right.$ ) en función de h es la siguiente:

$$
\mathrm{V}(\mathrm{h})=0.5 \cdot \mathrm{m} \cdot \mathrm{h}^{2}+\mathrm{n} \cdot \mathrm{h}
$$

donde $\mathrm{m}=-8.3143 \mathrm{y} \mathrm{n}=736$.

Por otra parte, para obtener la derivada del volumen (en $\mathrm{cm}^{3} / \mathrm{s}$ ) se usa la regla de la cadena:

$$
\begin{aligned}
\dot{V}(h) & =\frac{d V(h)}{d t}=\frac{d V(h)}{d h} \cdot \frac{d h}{d t}=\frac{d\left(0.5 \cdot m \cdot h^{2}+n \cdot h\right)}{d h} \cdot \dot{h} \\
& =(m \cdot h+n) \cdot \dot{h}=(-8.3143 \cdot h+736) \cdot \dot{h}
\end{aligned}
$$

\subsubsection{Caudal de entrada}

Se ha obtenido el caudal de la bomba para diferentes frecuencias, que ha sido aproximado mediante el polinomio de segundo grado en (5), que da el caudal de la bomba $\left(\mathrm{en}^{3} / \mathrm{s}\right)$ para un valor de frecuencia u dado. En la figura 4 se muestra dicho ajuste polinómico.

$$
\mathrm{q}_{\mathrm{e}}(\mathrm{u})=-0.0195 \cdot \mathrm{u}^{2}+5.61 \cdot \mathrm{u}-29.81
$$

\subsubsection{Caudal de salida}

El caudal de salida total es igual a la suma de los caudales de salida por cada válvula, una multivuelta y otra todo/nada. El caudal de salida por cada válvula depende de la altura $\mathrm{h}$; en concreto, de forma proporcional a la raíz cuadrada de h. El caudal de salida total vendría dado por la expresión (6).

$$
\begin{gathered}
q_{s}=q_{s 1}+q_{s 2}= \\
=\sqrt{\alpha_{1} \cdot h+\beta_{1}}+\sqrt{\alpha_{2} \cdot h+\beta_{2}}
\end{gathered}
$$

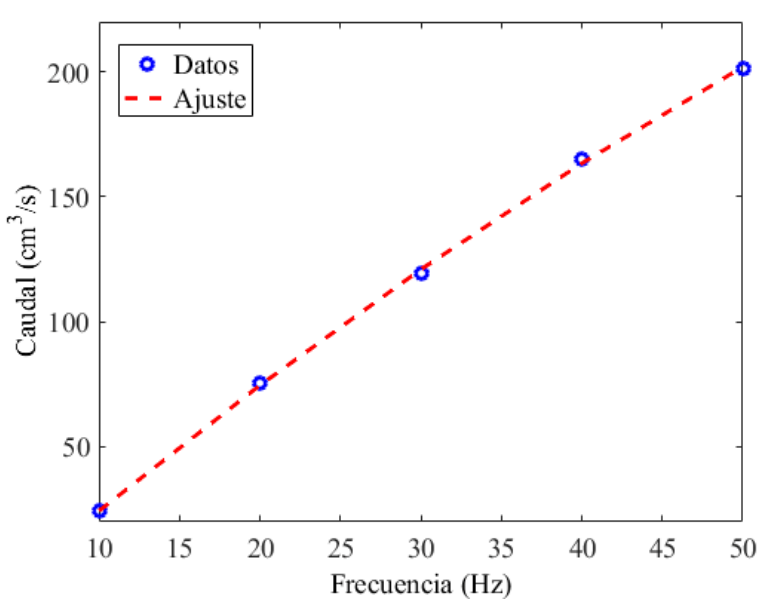

Figura 4: Ajuste polinomial de $\mathrm{q}_{\mathrm{e}}$

Para cada válvula, los parámetros $\alpha$ y $\beta$ se determinan a partir de datos experimentales obtenidos al vaciar el tanque trapezoidal por la válvula correspondiente. La expresión del caudal de salida en función del nivel de agua obtenida es:

$\mathrm{q}_{\mathrm{s}}=\sqrt{87.92 \cdot \mathrm{h}+602.73}+\sqrt{80.13 \cdot \mathrm{h}+888.22}$

En la figura 5 se muestran los datos de vaciado y su ajuste en simulación para la válvula multivuelta abierta a tope (arriba), y para la válvula todo-nada (debajo). Para cada válvula se han hecho dos experiencias similares. Se toman como valores de $\alpha$ y $\beta$ los valores promedios de ambas experiencias.

\subsubsection{Modelo no lineal completo}

A partir de las expresiones de los términos anteriores se llega al siguiente modelo no lineal completo del nivel del tanque trapezoidal en función de la frecuencia del variador:

$$
\begin{gathered}
\dot{h}=\frac{\left(-0.0195 \cdot u^{2}+5.61 \cdot u-29.8123\right)}{(-8.3143 \cdot h+736)} \\
-\frac{(\sqrt{87.92 \cdot h+602.73}+\sqrt{80.13 \cdot h+888.22})}{(-8.3143 \cdot h+736)}
\end{gathered}
$$

Este modelo se ha implementado en Simulink usando básicamente un bloque "Fcn" que implementa la ecuación (8) recibiendo u y h como entradas y dando la derivada de $h$, y un bloque integrador para obtener $\mathrm{h}$ a partir de su derivada. Para validar el modelo se realizó una experiencia con la frecuencia a $25 \mathrm{~Hz}$ y tras llegar a un estacionario se dio un salto a $30 \mathrm{~Hz}$. En la figura 6 se muestran los datos experimentales y los obtenidos en simulación con el modelo anterior. 
Aunque parten de un valor inicial algo diferente (unos pocos milímetros), el ajuste es bastante bueno.
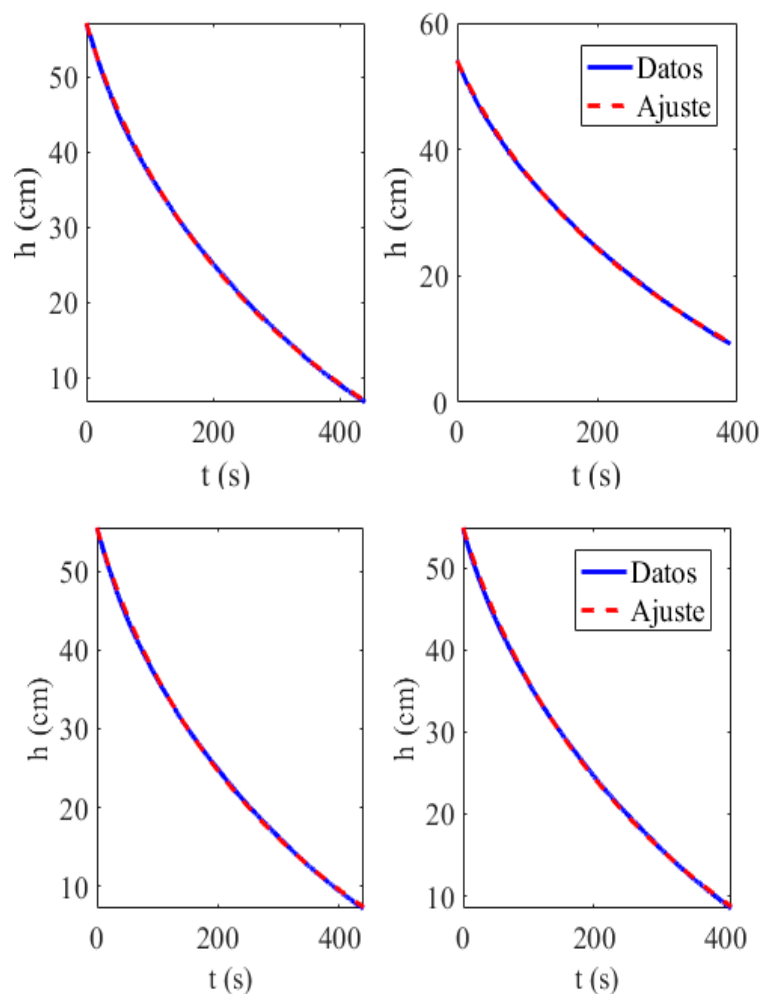

Figura 5: Datos de vaciado y ajuste

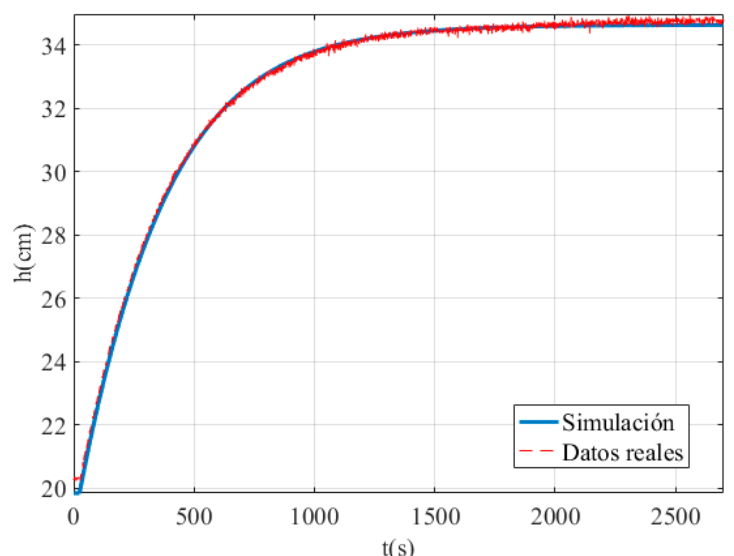

Figura 6: Validación del modelo no lineal

\subsection{LINEALIZACIÓN DEL MODELO NO LINEAL}

Para linealizar el modelo, es necesario definir un punto de equilibrio, definir variables incrementales en torno a dicho punto de equilibrio $\mathrm{y}$, por último, aplicar las series de Taylor para dichas variables. El polinomio de Taylor para la función $\dot{h}=f(h, u) d e$ orden "n" alrededor del punto $(\mathrm{u} 0, \mathrm{~h} 0)$ es el siguiente:

$\dot{h}=\dot{h}_{0}+\frac{\partial f(\mathrm{u} 0, \mathrm{~h} 0)}{\partial h}(h-h 0)+\frac{\partial f(\mathrm{u} 0, \mathrm{~h} 0)}{\partial u}(u-u 0)+$ $+\frac{1}{n !} \frac{\partial^{n} f(\mathrm{u} 0, \mathrm{~h} 0)}{\partial h}(h-h 0)^{n}+\frac{1}{n !} \frac{\partial^{n} f(\mathrm{u} 0, \mathrm{~h} 0)}{\partial u}(u-u 0)^{n}$

Utilizando sólo los términos de primer orden, el modelo linealizado es equivalente al polinomio de grado 1 de la serie anterior:

$$
\begin{gathered}
\dot{h}-\dot{h}_{0}=\frac{\partial f(\mathrm{u} 0, \mathrm{~h} 0)}{\partial h}(h-h 0)+\frac{\partial f(\mathrm{u} 0, \mathrm{~h} 0)}{\partial u}(u-u 0) \\
=k 1(h-h 0)+k 2(u-u 0)
\end{gathered}
$$

Los coeficientes $\mathrm{k} 1$ y $\mathrm{k} 2$ corresponden con las derivadas parciales en el punto de equilibrio de la función $\mathrm{h}=\mathrm{f}(\mathrm{h}, \mathrm{u})$, con respecto $\mathrm{a} h \mathrm{y} u$, respectivamente. Desarrollando ahora los términos diferencia en torno al punto de equilibrio como variables incrementales, se obtiene:

$$
\dot{\Delta h}=k 1 \cdot \Delta h+k 2 \cdot \Delta u
$$

Aplicando la transformada de Laplace a la ecuación anterior, se llega a la siguiente función de transferencia, donde el valor de los coeficientes $\mathrm{K}$ y $\tau$, depende del punto de equilibrio elegido.

$$
\frac{H(s)}{U(s)}=\frac{k 2}{s-k 1}=\frac{K}{\tau s+1}
$$

En la Figura 7 se muestra un análisis de los modelos linealizados en diferentes puntos de operación desde 20 a $37 \mathrm{~Hz}$. Se escoge ese rango porque fuera de él, el tanque se vacía o rebosa. Para cada frecuencia se calculan la ganancia y la constante de tiempo de la aproximación lineal de primer orden dada en (12). En la figura se muestran los valores de nivel estacionarios h0, constante de tiempo $\tau$ y ganancia $\mathrm{K}$.
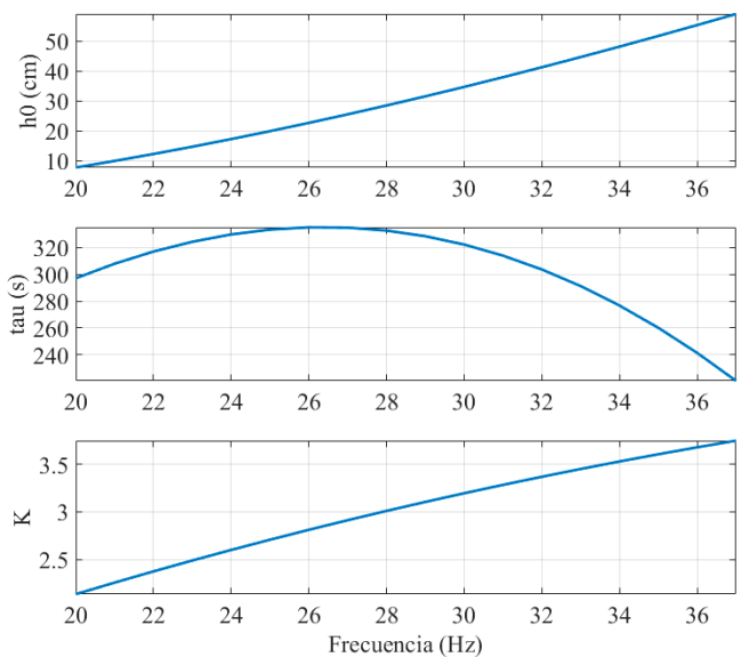

Figura 7: Parámetros del modelo linealizado según el punto de operación

En la Tabla 1 se detallan los parámetros de tres modelos lineales aproximados con los que se intenta abarcar todo el rango de operación. Teniendo en cuenta que el rango estacionario de altura del tanque 
va de 7.5 a $59 \mathrm{~cm}$, se divide el rango de nivel en 6 secciones de $8.5 \mathrm{~cm}$, lo que da 7 puntos, y se cogen los puntos 2, 4 y 6 . Por ello se proponen los puntos de operación para estimar los modelos correspondientes a las alturas siguientes: $16 \mathrm{~cm}$ (punto 1), $33 \mathrm{~cm}$ (punto 2) y $50 \mathrm{~cm}$ (punto 3 ). En la Figura 8 se muestra la respuesta ante escalón unitario de los tres modelos.

Tabla 1: Parámetros de tres modelos lineales

\begin{tabular}{|c|c|c|c|}
\hline $\mathrm{h}(\mathrm{cm})$ & Frecuencia $(\mathrm{Hz})$ & $\mathrm{K}$ & $\boldsymbol{\tau}$ \\
\hline $16(\mathrm{P} 1)$ & 23.53 & 2.549 & 327.55 \\
\hline $33(\mathrm{P} 2)$ & 29.48 & 3.148 & 325.84 \\
\hline $50(\mathrm{P} 3)$ & 34.53 & 3.569 & 268.17 \\
\hline
\end{tabular}

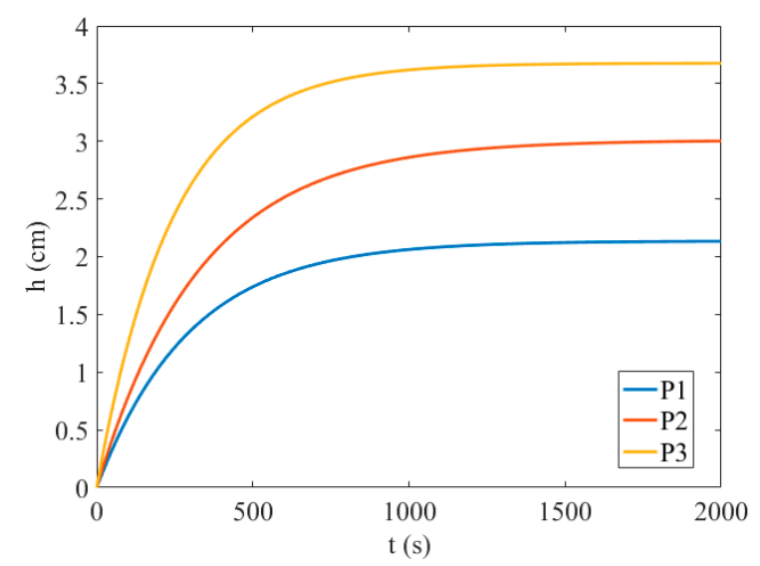

Figura 8: Respuesta escalón de los tres modelos lineales

\section{ESTRATEGIA DE CONTROL}

\subsection{CONTROL PI ADAPTATIVO CON GANANCIA PROGRAMADA}

El control adaptativo por ganancia programada consiste en ajustar de manera previa un controlador para diversos puntos de operación, y posteriormente, actualizar los parámetros de éste a partir de dichos diseños y según el punto de trabajo en el que esté el proceso. Hay diferentes esquemas de ganancia programada en la literatura. Uno de ellos equivale a tener varios controladores PI funcionando en paralelo donde sólo se escoge la salida de uno ellos en función de las condiciones de operación [1].

Por ejemplo, en la figura 9 se muestra un esquema muy simple de control PI por ganancia programada donde sólo habría 3 ajustes diseñados y funcionando en paralelo y donde se selecciona el más adecuado en función del valor de la referencia de lazo. Los otros dos entrarían en modo seguimiento de la salida final del controlador (teniendo en cuenta además el modelo de restricciones sobre la señal de control). En el presente trabajo se usa un controlador PI adaptativo similar donde se han ajustado 3 controladores PI, uno para cada modelo lineal identificado en el apartado anterior. Según el valor de la referencia de altura se decide la salida de cuál de ellos debe seleccionarse como señal de control final.

El controlador PI utilizado para cada lazo se implementa con una estructura I-P para evitar cambios bruscos en la señal de control ante cambios en la referencia. El controlador I-P no interactivo viene dado por la siguiente expresión:

$u(t)=K_{p}\left(-y(t)+\frac{1}{T_{i}} \int(r(t)-y(t)) d t\right)$

donde $u(t)$ es la señal de control, $r(t)$ la referencia, $\mathrm{y}(\mathrm{t})$ señal controlada, $\mathrm{Kp}$ es la ganancia proporcional y Ti es la constante de tiempo integral.

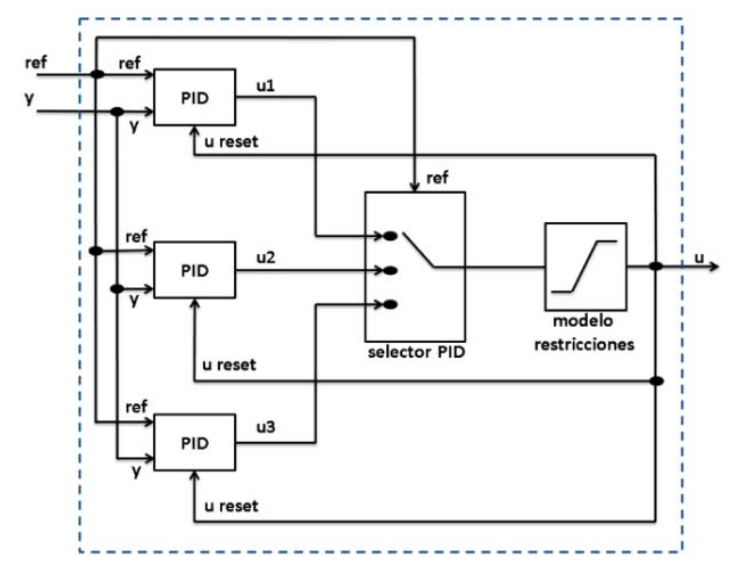

Figura 9: Esquema PI adaptativo

Esta ley de control continuo se implementa en una aproximación de tiempo discreto usando el algoritmo de Tustin. Las acciones proporcionales $\mathrm{P}(\mathrm{k})$ e integral $\mathrm{I}(\mathrm{k})$ de esta implementación en la k-ésima iteración se calculan como sigue:

$$
\begin{aligned}
& P(k)=-K_{p} \cdot y(k) \\
& I(k)=I(k-1)+K_{p i}(e(k)+e(k-1))
\end{aligned}
$$

La constante Kpi viene dada por (15), donde T es el tiempo de muestreo del controlador.

$$
K_{p i}=K_{p} \frac{T}{2 \cdot T_{i}}
$$

La señal de control sería la suma de estas dos acciones: $\mathrm{u}(\mathrm{k})=\mathrm{P}(\mathrm{k})+\mathrm{I}(\mathrm{k})$. Para implementar de forma sencilla el control por ganancia programada, todas estas variables $(\mathrm{P}(\mathrm{k}), \mathrm{I}(\mathrm{k}), \mathrm{u}(\mathrm{k}), \mathrm{Kp}, \mathrm{Kpi})$ son vectores del mismo tamaño que el de controladores en paralelo, tres en este caso. Mediante una sentencia if que evalúa el valor de la referencia, se decide qué controlador debe estar activo, obteniendo así la señal de control final $\mathrm{u}^{*}(\mathrm{k})$.

Para hacer frente al problema de wind-up, se implementa un mecanismo anti-windup utilizando un 
modelo de restricciones donde se consideran las saturaciones de la señal de control [6]. Cuando la señal de control final $\mathrm{u}^{*}(\mathrm{k})$ está fuera de sus límites, este mecanismo actualiza el término integral $\mathrm{I}(\mathrm{k})$ para que la salida del controlador no exceda dicho límite. El mecanismo se muestra en (16).

$$
\begin{aligned}
& \text { if } u^{*}(k)>u_{\max } \\
& \quad I(k)=u_{\text {max }}-P(k) \\
& \text { elseif } u^{*}(k)<u_{\text {min }} \\
& \quad I(k)=u_{\text {min }}-P(k) \\
& \text { else } I(k)=u^{*}(k)-P(k) \\
& \text { end }
\end{aligned}
$$

Además, como $\mathrm{I}(\mathrm{k})$ es un vector, también se actualizan las acciones integrales de todos los demás controladores para asegurar el seguimiento de estos a la señal $\mathrm{u}^{*}(\mathrm{k})$ del controlador activo. Así se consigue que la transición entre controladores sea suave y sin cambios bruscos en la señal de control. Es por ello que en la sentencia else, aunque no se excedan los límites de control, se actualiza el vector $\mathrm{I}(\mathrm{k})$ de tal forma que el resto de controladores no activos sigan a la señal $u^{*}(\mathrm{k})$.

\subsection{SINTONÍA DE LOS CONTROLADORES PI}

Los controladores PI se ajustan en el dominio de la frecuencia usando un margen de fase de $45^{\circ}$ como especificación [10] en cada uno de los modelos lineales especificados en la Tabla 1. Los parámetros resultantes se muestran en la Tabla 2.

Si por ejemplo, se usase el tercer controlador (punto P3) para los tres modelos de la Tabla 1 (esto es, usar un PI convencional), las respuestas en lazo cerrado ante escalón unitario en la referencia tendrían diferentes características en cuanto a sobreimpulso, tiempo de establecimiento, etc., tal como se muestra en la Figura 10, donde se observa un aumento de estos parámetros para los puntos $\mathrm{P} 1$ y $\mathrm{P} 2$ en comparación con el punto P3, aunque no se un aumento excesivamente notable.

Mediante el control PI adaptativo propuesto, se pretende que haya una menor variación en dicha respuesta, independientemente del punto de operación seleccionado. En el control propuesto, la señal de referencia se usa para determinar qué controlador está activo: por ejemplo, si la referencia está por debajo de $24.5 \mathrm{~cm}$ se escoge el PI para el punto 1 (P1); si está por encima de $41.5 \mathrm{~cm}$ el controlador del punto $3(\mathrm{P} 3)$; y en caso contrario, el controlador intermedio del punto $2(\mathrm{P} 2)$.

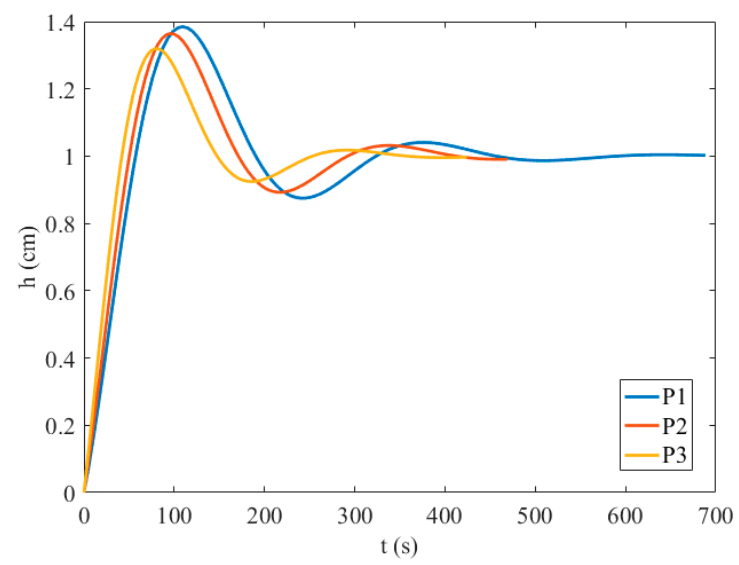

Figura 10: Respuesta escalón del sistema en lazo cerrado usando el PI del punto 3 sobre los tres modelos lineales

Tabla 2: Parámetros PI Adaptativo

\begin{tabular}{|c|c|c|}
\hline $\mathrm{h}(\mathrm{cm})$ & $\mathrm{Kp}$ & $\mathrm{Ti}$ \\
\hline $16(\mathrm{P} 1)$ & 2.48 & 26.9 \\
\hline $33(\mathrm{P} 2)$ & 2.01 & 26.76 \\
\hline $50(\mathrm{P} 3)$ & 1.77 & 22 \\
\hline
\end{tabular}

\subsection{IMPLEMENTACIÓN DEL CONTROL}

La implementación del controlador se realiza en un sistema de control distribuido Opto22, donde el nivel se mide mediante un sensor de presión de Wika el cual manda una señal proporciona 4-20 mA al módulo de entrada analógica del sistema Optpo22, también en corriente, y que posteriormente se escala en $\mathrm{cm}$. A su vez, como variable manipulada, se lleva al variador la salida analógica en corriente configurada del sistema Opto22 y que representa la frecuencia (en $\mathrm{Hz}$ ) de trabajo. La estrategia desarrollada se implementa en el entorno OptoControl y consiste básicamente en un bloque de ecuaciones en diferencias que se repite cada periodo de muestreo ( $1 \mathrm{~s}$ en este caso). Además, mediante OptoDisplay se ha creado un SCADA (Figura 11) para operar y configurar la planta experimental, así como guardar los datos de interés.

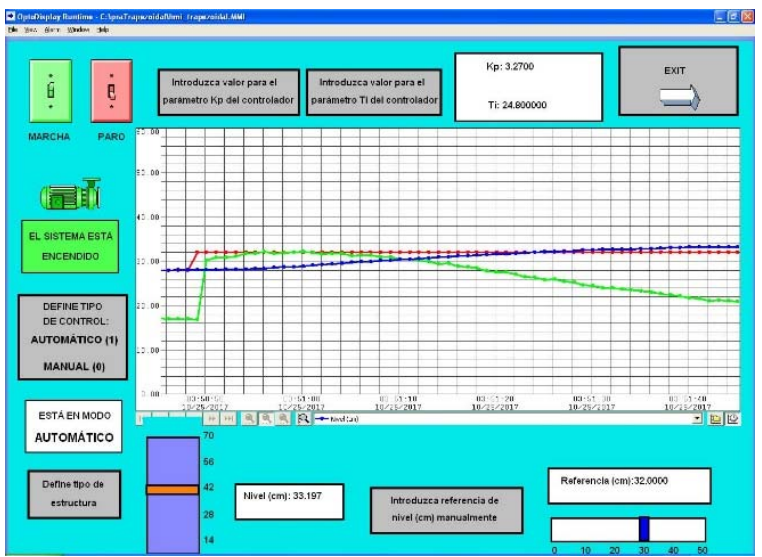

Figura 11: Pantalla principal del SCADA 


\section{4}

\section{RESULTADOS}

En este apartado se verifican los resultados tanto en simulación como experimentalmente del control PI adaptativo propuesto. Primero, partiendo de un punto de operación de unos $16 \mathrm{~cm}$ de altura, se lleva a cabo un salto escalón de 5 unidades en la referencia. En este tramo, el control ajustado para el punto P1 es el que funciona. Pasados unos $350 \mathrm{~s}$, se lleva a cabo un salto en la referencia a $45 \mathrm{~cm}$, para así movernos a un punto de operación alejado en el que debe funcionar el PI ajustado para el punto P3. Finalmente, pasados los $800 \mathrm{~s}$, se lleva a cabo otro salto escalón en la referencia de 5 unidades $(\mathrm{a} 50 \mathrm{~cm})$.

Las figuras 12 y 13 muestran la respuesta temporal de la altura del tanque y la señal de control, respectivamente, tanto en simulación como con la planta real. Se puede observar que las respuestas de la salida sistema tanto en torno al punto P1 como al punto P3 presentan características más similares que las mostradas en la Figura 9. Además, se observa una buena concordancia de los resultados experimentales con los obtenidos en simulación.

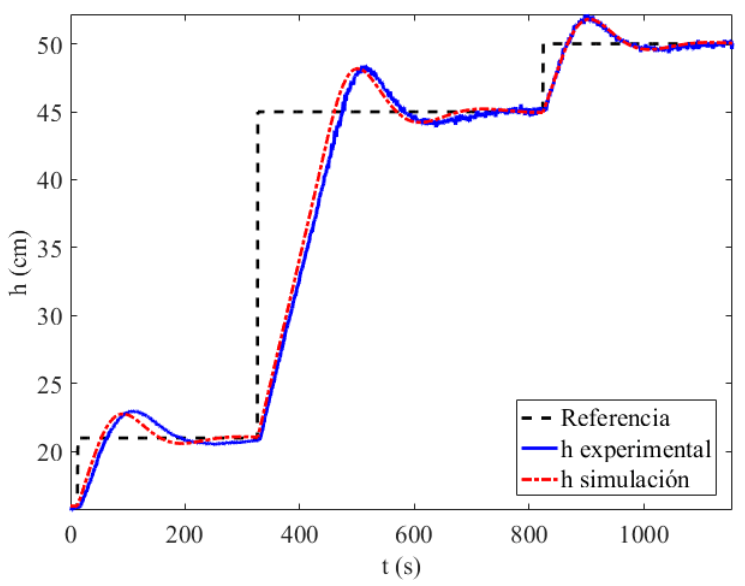

Figura 12: Respuesta temporal de la salida del sistema usando el control propuesto

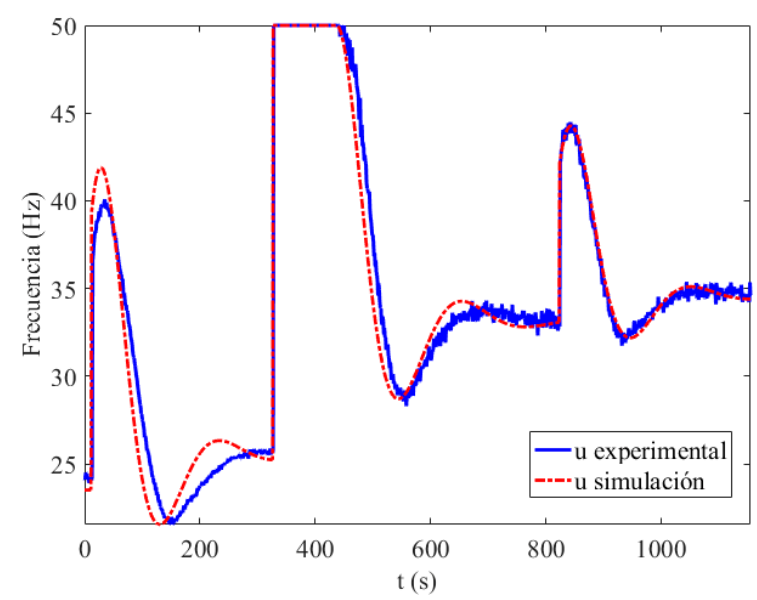

Figura 13: Respuesta temporal de la señal de control del sistema usando el control propuesto

\section{CONCLUSIONES}

En este trabajo se ha presentado cómo implementar de manera sencilla un control PI adaptativo por ganancia programada y su aplicación a un proceso experimental no lineal de control de nivel en un tanque de sección trapezoidal. Se ha obtenido un modelo no lineal del sistema a partir del cual llevar a cabo el ajuste de los controladores PI y que ha permitido realizar simulaciones cuya correspondencia con el proceso real son muy buenas. Las pruebas experimentales realizadas verifican el buen funcionamiento del sistema de control propuesto.

Además, el trabajo llevado a cabo ha permitido dotar a laboratorio de Control de Procesos de la Universidad de Córdoba de una nueva planta experimental para la enseñanza relacionada con control de nivel, controladores PI, sistemas no lineales y control por ganancia programada.

\section{Agradecimientos}

A la Universidad de Córdoba por el contrato de Personal Investigador en Formación (PIF) concedido a Manuel Lara.

\section{English summary}

\section{ADAPTIVE PI CONTROL BY GAIN SCHEDULING FOR A LEVEL CONTROL OF A TRAPEZOIDAL SECTION TANK}

\begin{abstract}
In this paper the problem of level control of a tank with trapezoidal section is addressed. The process is a non-linear system whose dynamic varies according to the operation point. An adaptive control structure is proposed based on a gain scheduling PI controller of easy implementation. The aforementioned controller is adjusted using methods in the frequency domain. The controller parameters are adjusted based on linearized models of the non-linear system around different operation points. Simulated and experimental results are compared and found in good agreement with a good system response.
\end{abstract}

Keywords: adaptive control, PI control, gain scheduling. 


\section{Referencias}

[1] Albertos, P., Mareels, I., (2010) Feedback and Control for Everyone. Springer.

[2] Aravind, P., Valluvan, M., Ranganathan, S., (2013) "Modelling and Simulation of Non Linear Tank", International Journal of Advanced Research in Electrical, Electronics and Instrumentation Engineering, vol. 2, issue 2, pp. 842-849.

[3] Bailey, R. L., Cederquist, A. L., Florek, J. J., Hart, D. L., \& Meitzler, A. H., (1978) "An IIEC-2 low-emission concept car", Society of Automotive Engineers, SAE Paper No. 780206

[4] Chandrasekar, Priya \& Lakshmi, P., (2013) "Passivity based level controller design applied to a nonlinear SISO system", International Conference on Green Computing, Communication and Conservation of Energy (ICGCE), pp. 392-396.

[5] Cook, J. A., Grizzle, J. W., \& Sun, J., (1996) The control handbook Engine control, editor William S. Levine, New York: IEEE Press, pp. 1261-1274.

[6] Garrido, J., Lara, M., Ruz, M., Vázquez, F., Alfaya, J. A., Morilla, F., (2018) "Decentralized PID control with inverted decoupling and superheating reference generation for efficient operation: Application to the Benchmark PID 2018", 3rd IFAC Conference on Advances in PID Control. Ghent, Belgium, pp. 710-715.

[7] Gireesh, N., Sreenivasulu, G., (2014) "Comparison of PI Controller Performances for a Conical Tank Process using different tuning methods", International Conference on Advances in Electrical Engineering.

[8] Gregory, P. C., (1959). Proceedings of the self adaptive flight control systems symposium, Wright-Patterson AFB. OH. WADC Technical Report Vol. 59 no. 49.

[9] Kala, H., Aravind, P., Valluvan, M., (2013) "Comparative Analysis of Different Controller for Nonlinear Level Control Process", IEE Conference on Information and Communication Technologies.

[10] Morilla, F., Dormido, S., (2000) "Methodologies for the tuning of PID Controllers in the frequency domain", Proceedings of IFAC Workshop on Digital
Control. Past, present and future of PID Control.

[11] Paddison, F. C., (1982). The Talos control system, Johns Hopkins APL Technical Digest, 3(2), 154-156.

[12] Rajesh, T., Arun jayakar, S., Siddharth, S. G., (2014) "Design and Implementation Of IMC Based PID Controller for Conical Tank Level Control Process", International Journal of Innovative Research in Electrical, Electronics, Instrumentation and Control Engineering, vol. 2 , issue 9 .

[13] Valarmathi,R., Theerthagiri,P.R., Rakeshkumar, S., (2013) "Design and Analysis of Genetic Algorithm Based Controllers for Non Linear Liquid Tank System", IEEE International Conference On Advances In Engineering, Science and Management.

[14] Vijula, D. A., Vivetha, K., Gandhimathi, K., Praveena, T., (2014) "Model based Controller Design for Conical Tank System", International Journal of Computer Applications, vol. 85, no. 12, pp. 8-11.

[15] Warier, S., Venkatesh, S., (2012) "Design of Controllers based on MPC for a Conical Tank System", International Conference On Advances In Engineering, Science and Managment.

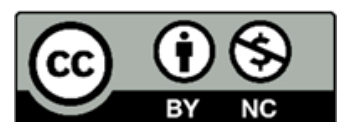

(C) 2018 by the authors. Submitted for possible open access publication under the terms and conditions of the Creative Commons Attribution CC-BY-NC 3.0 license (https://creativecommons.org/licenses/by-nc/3.0). 